\title{
Early life multiple exposures and child cognitive function: A multi-centric birth cohort study in six European countries ${ }^{\text {मै }}$
}

Jordi Julvez $^{\text {a,b,c, *,1 }}$, Mónica López-Vicente ${ }^{\text {b,c,d,e,1 }}$, Charline Warembourg b,c,d , Lea Maitre ${ }^{\text {b,c,d }}$, Claire Philippat $^{\mathrm{f}}$, Kristine B. Gützkow ${ }^{\mathrm{g}}$, Monica Guxens ${ }^{\mathrm{b}, \mathrm{c}, \mathrm{d}, \mathrm{e}}$, Jorunn Evandt ${ }^{\mathrm{g}}$, Sandra Andrusaityte ${ }^{\mathrm{h}}$, Miguel Burgaleta ${ }^{\mathrm{i}}$, Maribel Casas ${ }^{\mathrm{b}, \mathrm{c}, \mathrm{d}}$, Leda Chatzi ${ }^{\mathrm{j}, \mathrm{k}}$, Montserrat de Castro $^{\text {b,c,d, David Donaire-González }}{ }^{\text {b,c,d,l }}$, Regina Gražulevičienè ${ }^{\text {h }}$, Carles Hernandez-Ferrer ${ }^{\mathrm{b}, \mathrm{c}, \mathrm{d}}$, Barbara Heude ${ }^{\mathrm{m}}$, Rosie Mceachan ${ }^{\mathrm{n}}$, Mark Mon-Williams ${ }^{\mathrm{n}}$, Mark Nieuwenhuijsen ${ }^{\text {b, c, d }}$, Oliver Robinson ${ }^{\circ}$, Amrit K. Sakhi ${ }^{\mathrm{g}}$, Nuria Sebastian-Galles ${ }^{\mathrm{i}}$, Remy Slama ${ }^{\mathrm{f}}$, Jordi Sunyer ${ }^{\mathrm{b}, \mathrm{c}, \mathrm{d}}$, Ibon Tamayo-Uria ${ }^{\mathrm{b}, \mathrm{c}, \mathrm{d}, \mathrm{p}}$, Cathrine Thomsen ${ }^{\mathrm{g}}$, Jose Urquiza $^{\mathrm{b}, \mathrm{c}, \mathrm{d}}$, Marina Vafeiadi ${ }^{\mathrm{j}}$, John Wright ${ }^{\mathrm{n}}$, Xavier Basagaña ${ }^{\mathrm{b}, \mathrm{c}, \mathrm{d}}$, Martine Vrijheid ${ }^{\mathrm{b}, \mathrm{c}, \mathrm{d}}$

a Institut d'Investigació Sanitària Pere Virgili (IISPV), Hospital Universitari Sant Joan de Reus, Reus (Tarragona), Catalonia, Spain

${ }^{\mathrm{b}}$ Barcelona Institute for Global Health (ISGlobal), Barcelona, Catalonia, Spain

${ }^{\mathrm{c}}$ CIBER Epidemiologia y Salud Pública (CIBERESP), Instituto de Salud Carlos III, Madrid, Spain

${ }^{\mathrm{d}}$ Universitat Pompeu Fabra, Barcelona, Catalonia, Spain

${ }^{\mathrm{e}}$ Department of Child and Adolescent Psychiatry/Psychology, Erasmus University Medical Centre-Sophia Children's Hospital, PO Box 2060, 3000 CB, Rotterdam, the Netherlands

${ }^{\mathrm{f}}$ Team of Environmental Epidemiology Applied to Reproduction and Respiratory Health, Institute for Advanced Biosciences (IAB), Inserm U1209, CNRS UMR 5309, Université Grenoble Alpes, 38000, Grenoble, France

${ }^{g}$ Department of Environmental Health, Norwegian Institute of Public Health, Oslo, Norway

${ }^{\text {h }}$ Vytauto Didziojo Universitetas (VDU), Kaunus, Lithuania

${ }^{\mathrm{i}}$ Center for Brain and Cognition, Department of Technology, Universitat Pompeu Fabra, Roc Boronat 138, 08018, Barcelona, Catalonia, Spain

${ }^{\mathrm{j}}$ Department of Social Medicine, Faculty of Medicine, University of Crete, Heraklion, Greece

${ }^{\mathrm{k}}$ Department of Preventive Medicine, Keck School of Medicine, University of Southern California, Los Angeles, CA, USA

${ }^{1}$ Mary MacKillop Institute for Health Research, Australian Catholic University, Melbourne, Victoria, Australia

${ }^{\mathrm{m}}$ Universite de Paris, Centre for Research in Epidemiology and Statistics (CRESS), INSERM, INRAE, Paris, France

${ }^{\mathrm{n}}$ Bradford Institute for Health Research, Bradford Teaching Hospitals NHS Foundation Trust, Bradford, UK

${ }^{\circ}$ MRC Centre for Environment and Health, School of Public Health, Imperial College London, UK

p Division of Immunology and Immunotherapy, Cima Universidad de Navarra and "Instituto de Investigación Sanitaria de Navarra (IdISNA)", Pamplona, Spain

\section{A R T I C L E I N F O}

\section{Keywords:}

Environmental epidemiology

Exposome

Chemical biomarkers

Birth cohort study

Neurodevelopment

Neuropsychological development

\begin{abstract}
A B S T R A C T
Epidemiological studies mostly focus on single environmental exposures. This study aims to systematically assess associations between a wide range of prenatal and childhood environmental exposures and cognition. The study sample included data of 1298 mother-child pairs, children were 6-11 years-old, from six European birth cohorts. We measured 87 exposures during pregnancy and 122 cross-sectionally during childhood, including air pollution, built environment, meteorology, natural spaces, traffic, noise, chemicals and life styles. The measured cognitive domains were fluid intelligence (Raven's Coloured Progressive Matrices test, CPM), attention (Attention Network Test, ANT) and working memory (N-Back task). We used two statistical approaches to assess associations between exposure and child cognition: the exposome-wide association study (ExWAS) considering each exposure independently, and the deletion-substitution-addition algorithm (DSA) considering all exposures simultaneously to build a final multiexposure model. Based on this multiexposure model that included the exposure variables selected by ExWAS and DSA models, child organic food intake was associated with higher fluid intelligence $(\mathrm{CPM})$ scores (beta $=1.18 ; 95 \% \mathrm{CI}=0.50,1.87$ ) and higher working memory $(\mathrm{N}-\mathrm{Back})$ scores
\end{abstract}

\footnotetext{
This paper has been recommended for acceptance by Payam Dadvand.

* Corresponding author. Institut d'Investigació Sanitària Pere Virgili (IISPV), Hospital Universitari Sant Joan de Reus, Reus, Catalonia, Spain.

E-mail address: jordi.julvez@iispv.cat (J. Julvez).

$1 \mathrm{JJ}$ and ML-V share first authorship.
} 
$(0.23 ; 0.05,0.41)$, and child fast food intake $(-1.25 ;-2.10,-0.40)$, house crowding $(-0.39 ;-0.62,-0.16)$, and child environmental tobacco smoke (ETS) $(-0.89 ;-1.42,-0.35)$, were all associated with lower CPM scores. Indoor $\mathrm{PM}_{2.5}$ exposure was associated with lower N-Back scores $(-0.09 ;-0.16,-0.02)$. Additional associations in the unexpected direction were found: Higher prenatal mercury levels, maternal alcohol consumption and child higher perfluorooctane sulfonic acid (PFOS) levels were associated with better cognitive performance; and higher green exposure during pregnancy with lower cognitive performance. This first comprehensive and systematic study of many prenatal and childhood environmental risk factors suggests that unfavourable child nutrition, family crowdedness and child indoor air pollution and ETS exposures adversely and cross-sectionally associate with cognitive function. Unexpected associations were also observed and maybe due to confounding and reverse causality.

$\begin{array}{ll}\text { Abbreviations list } \\ \text { ANT } & \text { Attention Network Test } \\ \text { BiB } & \text { Born in Bradford } \\ \text { BPA } & \text { Bisphenol-A } \\ \text { CI } & \text { Confidence Interval } \\ \text { CPM } & \text { Raven's Coloured Progressive Matrices test } \\ \text { DDE } & \text { Dichlorodiphenyldichloroethylene } \\ \text { DDT } & \text { Dichlorodiphenyldichloroethylene } \\ \text { DEHP } & \text { Di-EthylhexylPhthalate } \\ \text { DETP } & \text { Diethyl thiophosphate } \\ \text { DMTP } & \text { Dimethyl thiophosphate } \\ \text { DSA } & \text { Deletion-substitution-addition algorithm } \\ \text { EDEN } & \text { Étude des Déterminants pré et postnatals du } \\ & \text { développement et de la santé de l'Enfant } \\ \text { ETS } & \text { Environmental tobacco smoke } \\ \text { ExWAS } & \text { Exposome-wide association study } \\ \text { HCB } & \text { Hexachlorobenzene } \\ \text { HELIX } & \text { Human Early-Life Exposome } \\ \text { HRT-SE } & \text { Hit Reaction Time Standard error } \\ \text { INMA } & \text { INfancia y Medio Ambiente } \\ \text { KANC } & \text { Kaunus Cohort } \\ \text { KIDMED } & \text { Mediterranean diet in children } \\ \text { MEHHP } & \text { Mono-2-ethyl-5-hydroxyhexyl phthalate } \\ \text { MEHP } & \text { Mono-(2-ethylhexyl) phthalate } \\ & \end{array}$

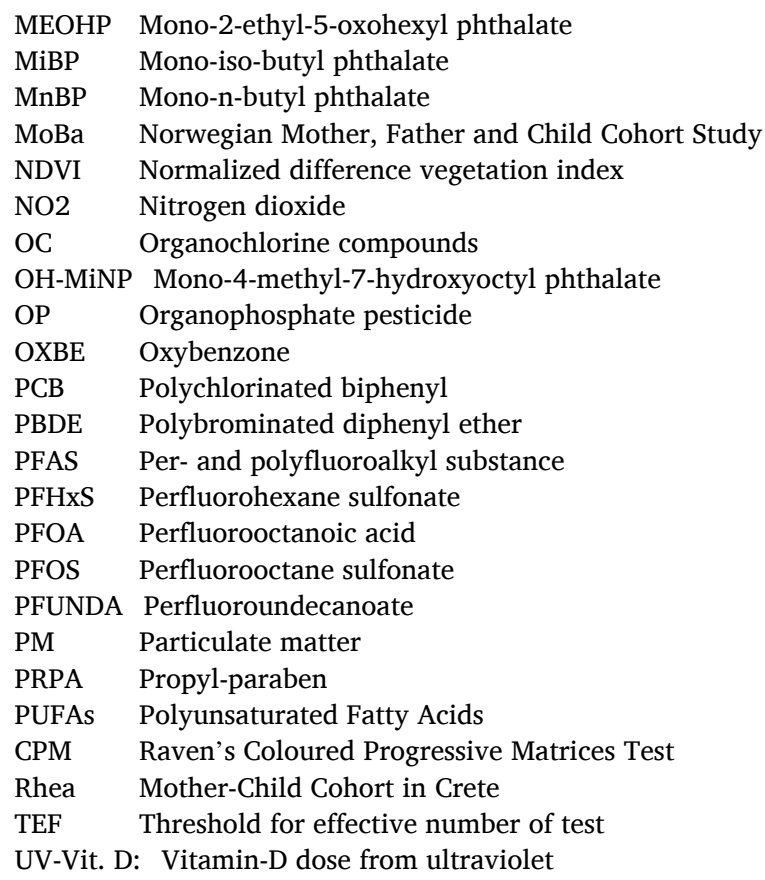

vulnerability at early-life exposure periods, such as pregnancy and early childhood. The epidemiological evidence is less clear for exposures of perfluoroalkyl substances (Forns et al., 2015), and non-persistent chemicals, such as phthalate metabolites (Olesen et al., 2017), and phenols (Braun et al., 2017). Additionally, exposure to ambient air pollutants (Sunyer et al., 2015) and road traffic noise (Weyde et al., 2017) during childhood have been reported to be adversely associated with neurodevelopment. Further, there are many other environmental factors associated with cognitive improvements, including living closer to green spaces (Dadvand et al., 2017) and in neighbourhoods with more facilities including parks and recreation centres (Bitsko et al., 2016), consuming a healthy diet (Cohen et al., 2016), and living within socioeconomically advantaged families and parents with higher education (Pearce et al., 2016).

Currently, most of the studies on environmental neuroepidemiology are based on single exposures (e.g., mercury) or exposure families (e.g., metals) (Debes et al., 2016; Braun et al., 2012; Forns et al., 2012; Roze et al., 2009; van Wendel de Joode et al., 2016; Hsieh et al., 2008; Forns et al., 2015; Olesen et al., 2017; Braun et al., 2017; Sunyer et al., 2015; Weyde et al., 2017; Dadvand et al., 2017). However, human cognitive development interacts concurrently with hundreds of environmental determinants during the lifespan (Grandjean and Landrigan, 2014). Indeed, the study of single exposures may increase selective reporting and be limited by co-exposure confounding (Vrijheid et al., 2014). Recently, a new approach is being developed in environmental 
epidemiology toward the concept of the human exposome, the totality of environmental exposure analogous to the genome. Thus, the exposome analytical approach aims to study a wide range of environmental exposures of an individual and to analyse the role of such environmental factors as multi-factorial risk factors of child neurodevelopment (Vrijheid et al., 2014). A few recent studies on child cognitive development used this new approach with a modest number of exposures (Steer et al., 2015; Calamandrei et al., 2020). In this study, the exposures were selected at the start of the HELIX project, because they were of concern for more than one of the health outcomes under study in the entire project (fetal and child growth, obesity and cardiometabolic outcomes, neurodevelopment, and respiratory outcomes) and because population exposure was widespread. Several statistical models were tested following simulation proceedings (Agier et al., 2016), and exposome-wide association study (ExWAS) analysis in combination with 'deletion-substitution-addition' (DSA) algorithm were the best fitted models to find relevant exposure determinants with a balanced type I and II statistical errors.

The present study aims to analyse simultaneously a set of 87 prenatal and 122 childhood exposures in relation to the cognitive function of 1298 children from six European birth cohorts (Vrijheid et al., 2014; Tamayo-Uria et al., 2019; Maitre et al., 2018; Haug et al., 2018). We used specific cross-culturally validated neuropsychological tests to assess fluid intelligence, attention function and working memory.

\section{Methods}

\subsection{Study participants}

Mother-child pairs were selected from six European birth cohorts included in the Human Early-Life Exposome (HELIX) project (Tamayo-Uria et al., 2019): BiB (Born in Bradford; United Kingdom), EDEN (Étude des Déterminants pré et postnatals du développement et de la santé de l'Enfant; France), INMA (INfancia y Medio Ambiente; Spain), KANC (Kaunus Cohort; Lithuania), MoBa (Norwegian Mother, Father and Child Cohort Study; Norway) and Rhea (Greece). A sub-cohort sample was selected to include 1298 children (BiB, $n=204$; EDEN, $n$ $=198$; INMA, $\mathrm{n}=221$; KANC, $\mathrm{n}=204 ; \mathrm{MoBa}, \mathrm{n}=272$; Rhea, $\mathrm{n}=199$ ) according to the following criteria of eligibility: a) primary school ages (6-11 years), b) stored pregnancy blood and urine samples available, c) complete address history, and d) no serious child health problems that may affect the clinical testing or the child safety (Maitre et al., 2018). The 1298 mother-child pairs were assessed by trained personnel according to the same harmonized protocol. The assessment included a maternal computer-based questionnaire, child computer-based cognitive tests and clinical examination. The mothers also completed a computer-based cognitive test. Biological samples from the child were also collected. The ethics committee for each cohort approved the consent form. We obtained written informed consent for all participants, signed by the parent.

\subsection{Exposure measurements}

The HELIX project aimed to assess the early-life exposome and a wide range of exposures were evaluated during pregnancy and childhood. In the present study, 87 maternal prenatal exposures and 122 childhood exposures (6-11 years old) were included. The complete exposome variable list with abbreviated names is shown in Supplementary Annex 1, Table A1.1.A; extended information with corresponding categories, and, with a summary description along with missing values (\%) out of 1298 mother-child pairs, is shown in Supplementary Annex 1, Table A1.1.B. There are publications regarding chemical assessment (Haug et al., 2018), questionnaires and general cohort description (Maitre et al., 2018) and correlations between exposure and time period (Tamayo-Uria et al., 2019).

In summary, we used cohorts' stored samples to measure chemical exposure during pregnancy. The chemicals were measured in cord blood, maternal serum, plasma, whole blood and urine. For the childhood chemical exposures, we newly collected child samples of serum, plasma, whole blood and urine following a common protocol in the involved cohorts (Haug et al., 2018). The chemicals measured in both periods (pregnancy and childhood) included organochlorines (polychlorinated biphenyls [PCBs] and organochlorine pesticides), polybrominated diphenyl ethers (PBDEs), perfluorinated alkylated substances (PFAS), metals, phthalate metabolites, phenols, organophosphate pesticide metabolites, and cotinine. When appropriate, the concentrations were standardized for lipids or creatinine (Haug et al., 2018).

The urban exposome was estimated after geo-coding the home address for the pregnancy period and the home and school addresses for the childhood period. Methods are fully described elsewhere (Tamayo-Uria et al., 2019; Robinson et al., 2018). The urban exposures were air pollution (e.g. $\mathrm{PM}_{2.5}, \mathrm{NO}_{2}$ ), natural spaces in the living area (green and blue spaces), meteorological conditions (outdoor temperature, atmospheric pressure, and humidity), markers of the built environment (e. g., population and facility densities and street connectivity) and road traffic and traffic noise. Additional exposures assessed at the home address by predictive modelling include water disinfection by-products (Nieuwenhuijsen et al., 2009) (for pregnancy only) and indoor pollutants (for childhood period only, based on questionnaire and in-house sensor data).

Life style factors were collected by questionnaires and included maternal smoking habits and alcohol use during pregnancy, maternal and child diet, child physical activity, child sleep and family social and economic capital during childhood. Prenatal life style variables were harmonized from previously collected data during pregnancy. Childhood life style variables were collected when children aged 6-11 years following the same protocol and questionnaires among cohorts, and were referring to the child's habits during the last year.

\subsection{Cognitive measurements}

Trained fieldwork technicians measured three cognitive domains in children using a battery of computer-based tests: fluid intelligence (Raven Coloured Progressive Matrices Test [CPM]), attention function (Attention Network Test [ANT]) and working memory (N-Back task). Complete outcome descriptions are provided in Supplementary Annex 2. The CPM comprised a total of 36 items and we used the total number of correct responses as the outcome. A higher CPM scoring indicates better fluid intelligence. Fluid intelligence is the ability to solve novel reasoning problems and depends only minimally on prior learning (Raven, 1998). For ANT, we used the outcome of hit reaction time standard error (HRT-SE), a measure of response speed consistency throughout the test. A high HRT-SE indicates highly variable reaction time during the attention task and is considered a measure of inattentiveness (Forns et al., 2014). As the main parameter of N-Back, we used d prime $\left(d^{\prime}\right)$, a measure derived from signal detection theory calculated by subtracting the z-score of the false alarm rate from the z-score of the hit rate. A higher $\mathrm{d}^{\prime}$ indicates more accurate test performance, i.e. better working memory (Forns et al., 2014). The N-Back task was also completed by the mothers. All examiners were previously trained following a standardized assessment protocol by the study expert psychologist. Furthermore, during the pilot phase, a coordinator visited each cohort site and checked for any potential error committed by the previously trained examiners. We used all the outcomes as cognitive gross scores and we adjusted them by child age in the regression models.

\subsection{Data pre-processing}

Exposures not assessed in more than 2 cohorts, or exposures with more than $70 \%$ of missing overall, were excluded. Finally, for sets of exposures that measure a very similar thing and that exhibit correlations 
higher than 0.9 , only one exposure representative of the group was included, as they were considered to measure the same thing and it is very difficult to separate the effect of one or the other. This concern variables of the outdoor environment calculated at different time point or different buffers. Time-varying exposures including air pollution and meteorology were averaged within different intervals: the whole pregnancy, the 1st, 2nd, and 3rd trimesters of pregnancy, the first year of life, and the year, the month, or the day before the measurement of cognitive functioning. The built environment and natural spaces were measured within different buffers: $100 \mathrm{~m}, 300 \mathrm{~m}$, or $500 \mathrm{~m}$. For each variable, the optimal transformation to approach normality was applied, or the variable was categorized if normality could not be achieved. Missing data for all exposures and confounders were imputed using the method of chained equations. A total of 20 imputed datasets were generated and used in all the analyses mentioned hereafter. Rubin's rule was used to aggregate the results from the 20 imputed datasets (White et al., 2011). For exposure biomarkers, values under the limit of detection were then imputed using single imputation distribution-based method (Jin et al., 2011). More details on the transformation used for each variable and its missing rate are provided in Supplementary Annex 1, Table A1.1.B.

\subsection{Statistical analyses}

The statistical methods were identified a priori through a series of simulation studies mimicking as closely as possible the situation expected with HELIX data (Agier et al., 2016). First, as a screening method, an exposome-wide association study (ExWAS) analysis was performed to obtain exposure-by-exposure estimations of the associations with cognitive function (CPM correct responses, ANT HRT-SE and N-Back d' scores), using multivariable linear regressions adjusting for confounders (Agier et al., 2016). A family wise error rate correction was used to account for multiple comparisons ( $5 \%$ divided by the effective number of tests, determined according to the correlation structure of the data) leading to corrected p-values equal to 0.001 and 0.0007 for the prenatal and childhood analyses respectively (Li et al., 2012). With a sample size of 1,200 , the agnostic EWAS analysis with control for multiple testing has a power of $80 \%$ to detect a 3 -point difference in a continuous outcome variable with a standard deviation of 15 (as in common neurodevelopment indexes). Then, we applied the iterative model search 'deletion-substitution-addition' (DSA) algorithm, a method that selects the exposures that are jointly associated with the outcome of interest (Sinisi and van der Laan, 2004). DSA uses cross-validation, which is subject to sampling error. Therefore, DSA was run 50 times, with no polynomial or interaction terms allowed, adjusting for confounders, and all the exposures that were selected in at least $5 \%$ of the runs were included in a final linear regression model (i.e., called thereafter 'multi-exposures model'). The $5 \%$ cut-off was arbitrary and a priori selected with the aim of reducing false positive findings. DSA was shown to provide a lower proportion of false positive associations than ExWAS (Agier et al., 2016). In conclusion, DSA was used in order to build the final multi-exposure regression models.

Both statistical methods (ExWAS and DSA) were performed separately for the prenatal and the childhood exposomes and were adjusted for potential confounders identified from a Directed Acyclic Graph drawn using DAGitty (Textor et al., 2011). For both time periods, the selected confounders included the cohort of inclusion (BiB, EDEN, INMA, KANC, MoBa, Rhea), maternal age (continuous), maternal education level (low, middle, high) as a proxy of socio-economic factors, trimester of the year of child conception (categorical variable), child age at cognitive examination (continuous) and child sex (Supplementary Annex 3). Other important health factors such as maternal obesity and diabetes during pregnancy were excluded due to the fact they were consider potential intermediate factors of the exposome and child neurodevelopment.

The estimates reported for continuous variables are expressed as an increase in interquartile range.
All analyses were run under R3.4.0 (The R Project for Statistical Computing, Vienna, Austria).

\subsection{Sensitivity analyses}

Several sensitivity analyses were performed for the multi-exposure models including: 1) complete case analyses, 2) analyses stratified by cohort, 3) analyses stratified by maternal education level, 4) analyses additionally adjusted for maternal perceived stress (Lee et al., 2015), maternal working memory (N-Back d' scores) (Forns et al., 2014) and maternal seafood intake.

\section{Results}

The mean child age was 8 years and varied by cohort $(\mathrm{BiB}=6.6$, EDEN $=10.8$, INMA $=8.8$, KANC $=6.5, \mathrm{MoBa}=8.5$, Rhea $=6.5$ ). There were more male children (54.7\%) than female children. 1011 children completed the three tests, the CPM and the ANT were completed by 1275 , the CPM and the N-back were completed by 1013 , and finally, 1017 participants completed the ANT and the N-back. The cognitive scores are presented by maternal and child characteristics in Table 1 . Generally, we observed better scores among EDEN, INMA and MoBa children and among children of older and highly educated mothers. Older children obtained better scores on all the cognitive tests (CPM correct responses [fluid intelligence], ANT HRT-SE [attention function or inattentiveness], N-Back d' [working memory]). Furthermore, boys performed better than girls on ANT HRT-SE. The cohort differences in the raw scores of the tests are basically attributed to cohort age differences (data not shown).

Exposome-wide associations (ExWAS) between prenatal and

Table 1

Child neuropsychological scores by selected confounders.

\begin{tabular}{|c|c|c|c|c|c|c|}
\hline & \multicolumn{2}{|c|}{$\begin{array}{l}\text { CPM, number } \\
\text { of correct } \\
\text { responses (fluid } \\
\text { intelligence, } \\
\text { Range: } 9 \text { to } 36 \text { ) }\end{array}$} & \multicolumn{2}{|c|}{$\begin{array}{l}\text { ANT, HRT-SE } \\
\text { (inattentiveness, } \\
\text { Range: } 81 \text { to } 676 \text { ) }\end{array}$} & \multicolumn{2}{|c|}{$\begin{array}{l}\text { 3-Back, d' } \\
\text { (working } \\
\text { memory, } \\
\text { Range: } 1.57 \text { to } \\
\text { 3.92) }\end{array}$} \\
\hline & $\mathrm{N}$ & Median & $\mathrm{N}$ & Median & $\mathrm{N}$ & Median \\
\hline \multicolumn{7}{|l|}{ Cohorts } \\
\hline $\mathrm{BiB}, \mathrm{UK}$ & 203 & $21 *$ & 201 & $355.1 *$ & 176 & $0.78^{*}$ \\
\hline EDEN, France & 195 & 33 & 187 & 237.7 & 184 & 1.90 \\
\hline INMA, Spain & 220 & 30 & 219 & 256.5 & 217 & 1.39 \\
\hline KANC, Lithuania & 204 & 23 & 204 & 367.7 & - & - \\
\hline MoBa, Norway & 272 & 31 & 272 & 248.7 & 263 & 1.39 \\
\hline Rhea, Greece & 198 & 20 & 198 & 340.9 & 179 & 0.80 \\
\hline \multicolumn{7}{|l|}{ Maternal education } \\
\hline Low & 181 & $23^{*}$ & 178 & $330.0^{*}$ & 153 & $0.84 *$ \\
\hline Middle & 441 & 26 & 436 & 305.1 & 345 & 1.39 \\
\hline High & 670 & 29 & 667 & 291.3 & 521 & 1.39 \\
\hline \multicolumn{7}{|c|}{ Maternal age (years) } \\
\hline$<27$ & 326 & $24 *$ & 322 & $339.3^{*}$ & 216 & $1.03^{*}$ \\
\hline$\geq 27 \&<31$ & 322 & 28 & 319 & 291.4 & 256 & 1.39 \\
\hline$\geq 31 \&<34$ & 321 & 29 & 319 & 280.5 & 272 & 1.29 \\
\hline$\geq 34$ & 323 & 28 & 321 & 294.1 & 275 & 1.39 \\
\hline \multicolumn{7}{|c|}{ Trimester of conception } \\
\hline Jan-March & 415 & 27 & 415 & 305.1 & 331 & 1.39 \\
\hline April-June & 261 & 27 & 259 & 302.2 & 217 & 1.12 \\
\hline July-Sept & 280 & 26 & 279 & 298.0 & 201 & 1.39 \\
\hline Oct-Dec & 336 & 28 & 328 & 294.1 & 270 & 1.29 \\
\hline \multicolumn{7}{|l|}{ Child age (years) } \\
\hline$<6.5$ & 325 & $21^{*}$ & 325 & $370.5^{*}$ & 171 & $0.80^{*}$ \\
\hline$\geq 6.5 \&<8$ & 318 & 24 & 316 & 332.7 & 221 & 0.80 \\
\hline$\geq 8 \&<9$ & 320 & 30 & 320 & 260.8 & 311 & 1.39 \\
\hline$\geq 9$ & 329 & 32 & 320 & 233.7 & 316 & 1.71 \\
\hline \multicolumn{7}{|l|}{ Child sex } \\
\hline Male & 707 & 27 & 703 & $293.5^{*}$ & 564 & 1.17 \\
\hline Female & 585 & 27 & 578 & 309.7 & 455 & 1.39 \\
\hline
\end{tabular}

Data are medians. Kruskal-Wallis equality-of-populations rank tests. * Pvalue $<0.10$. 
childhood exposures and CPM scores are shown in Table 2. This method gave us a first picture of the association patterns observed with all the exposures analysed independently. Child organic food consumption and childhood house crowding passed the p-value correction threshold. Table 3 and Table 4 show the ExWAS results of the ANT HRT-SE and NBack d' scores, and no exposure association passed the p-value correction threshold. Fig. 1 gives a graphic representation of the same ExWAS results, all the exposures with a p-value below 0.05 are labelled.

We further applied DSA analyses for exposure variable selection based on jointly association patterns. Tables $2-4$ also show the results of the DSA analyses. In Table 2, prenatal mercury was selected by DSA on $6 \%$ of the runs (minimum condition was set to $5 \%$ of the runs), although higher exposure was associated with higher CPM scores in the single regression model shown in the same table. Child organic food consumption was selected by DSA on $94 \%$ of the runs and higher intake was associated with higher CPM scores. Childhood house crowding was selected by DSA (66\%) and higher crowding showed a reduction of CPM scores. DSA method also selected child fast-food intake (58\%), PFOS concentration (20\%), and ETS exposure (44\%). Increments of all of them, except PFOS, were associated with a reduction of CPM scores.

In relation to ANT HRT-SE (Table 3), prenatal maternal alcohol consumption (yes/no) and green space exposure according to Normalized Difference Vegetation Index (NDVI) were selected by DSA, 26\% and $46 \%$, respectively. However, prenatal alcohol consumption was associated with lower inattentiveness scores and more green space exposure was associated with higher inattentiveness scores. Prenatal DMTP concentration (organophosphate pesticide) was selected by $6 \%$ of DSA runs. No childhood exposures were selected by DSA in Table 3.

For N-Back d' scores (Table 4), child indoor air pollution (indoor $\mathrm{PM}_{2.5}$ ) was selected by DSA with a small percentage of the runs (6\%), and the association showed a score reduction. Finally, organic food intake was also selected (12\%) and the association showed an increase of the N-Back d' score. No prenatal exposures were selected by DSA.

Table 5 presents a multi-exposure regression model per each outcome and keeping prenatal and childhood exposures in separate models. This model included all the exposure variables selected in at least $5 \%$ of the DSAs. The association coefficients were similar to the previous single exposure models shown in Tables 2-4, and all the selected exposure variables, except DMTP, retained their statistically significant associations with the CPM, ANT and N-Back outcomes. Child organic food intake was the only exposure associated with two outcomes (CPM scores and N-Back d') at the same time.

In the sensitivity analyses, repeating the multi-exposure models with complete cases (Supplementary Table A4.1.A. and Table A4.1.B.) or adjusting models for maternal perceived stress (Supplementary Table A4.2.) and maternal N-Back d' scores (Supplementary Table A4.3.), we did not observe differences with the previous findings. All the exposure variables tended to show a similar association patterns by cohort (Supplementary Figures A4.1. and A4.2.), with the exception of child PFOS concentrations which showed some heterogeneity in the association with CPM (Supplementary Figure A4.2.). In relation to the exposures with 'un-expected' association directions, we stratified the final models by maternal education (high and medium-low). Alcohol consumption and PFOS concentration only were associated with child cognitive outcomes in the high education group, meanwhile prenatal green space exposure (NDVI-100) association only occurred in lower education. However, prenatal mercury and DMTP exposures showed similar coefficients in both education groups (Supplementary Table A4.4.). All these variables tended to show higher exposure levels in the higher maternal education group (data not shown). The findings with mercury exposures were unchanged after adjusting for maternal seafood intake (data not shown).

Finally, we tested the linearity of the multi-exposure associations by plotting the residuals versus the predicted values and we repeated the

Table 2

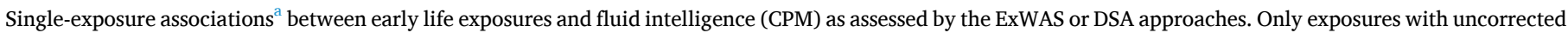
p-values $<0.05$ or selected from DSA are shown.

\begin{tabular}{|c|c|c|c|c|c|c|}
\hline & \multicolumn{5}{|l|}{ ExWAS } & \multirow{2}{*}{$\frac{\text { DSA }}{\text { Frequency (\%) of selection }}$} \\
\hline & $\mathrm{p}$ & Adjustec & ient $(95 \%$ & & Significant after p-value correction? & \\
\hline \multicolumn{5}{|l|}{ Prenatal Exposome } & \multicolumn{2}{|l|}{$\mathrm{TEF}=0.001$} \\
\hline Facility density $(300 \mathrm{~m})$ & 0.013 & 0.509 & $(0.109$ & $0.910)$ & No & 2 \\
\hline Mercury & 0.014 & 0.611 & $(0.122$ & 1.099) & No & 6 \\
\hline PFOA & 0.024 & 0.451 & $(0.061$ & $0.842)$ & No & - \\
\hline PFhXS & 0.039 & 0.381 & $(0.019$ & $0.743)$ & No & - \\
\hline NDVI (100 m) & 0.044 & -0.722 & $(-1.424$ & $-0.021)$ & No & - \\
\hline \multicolumn{5}{|l|}{ Childhood Exposome } & \multicolumn{2}{|l|}{$\mathrm{TEF}=0.0007$} \\
\hline Organic food intake & $<0.001$ & NA & NA & NA & Yes & 94 \\
\hline 2nd tertile vs. 1 st tertile & $<0.001$ & 1.255 & $(0.632$ & $1.878)$ & - & - \\
\hline 3rd tertile vs. 1 st tertile & $<0.001$ & 1.332 & $(0.642$ & 2.022) & - & - \\
\hline House crowding & 0.001 & -0.412 & $(-0.646$ & $-0.178)$ & Yes & 66 \\
\hline Fastfood intake & 0.002 & NA & NA & NA & No & 58 \\
\hline 2nd tertile vs. 1 st tertile & 0.729 & 0.095 & $(-0.444$ & $0.635)$ & - & - \\
\hline 3rd tertile vs. 1 st tertile & 0.001 & -1.434 & $(-2.294$ & $-0.575)$ & - & - \\
\hline Lead & 0.003 & -0.436 & $(-0.724$ & $-0.149)$ & No & 2 \\
\hline ETS & 0.003 & -0.816 & $(-1.354$ & $-0.279)$ & No & 44 \\
\hline PFOS & 0.006 & 0.501 & $(0.143$ & $0.860)$ & No & 20 \\
\hline Soda intake & 0.022 & NA & NA & NA & No & - \\
\hline 2nd tertile vs. 1 st tertile & 0.061 & -0.530 & $(-1.083$ & $0.023)$ & - & - \\
\hline 3rd tertile vs. 1 st tertile & 0.007 & -0.859 & $(-1.486$ & $-0.231)$ & - & - \\
\hline Indoor benzene & 0.014 & -0.438 & $(-0.789$ & $-0.087)$ & No & - \\
\hline Arsenic & 0.018 & 0.515 & $(0.088$ & $0.942)$ & No & - \\
\hline PCB 180 & 0.031 & 0.509 & $(0.047$ & $0.972)$ & No & - \\
\hline DETP & 0.034 & 0.419 & $(0.031$ & $0.806)$ & No & - \\
\hline PFOA & 0.036 & 0.348 & $(0.023$ & $0.674)$ & No & - \\
\hline PCB 170 & 0.037 & 0.456 & $(0.028$ & $0.884)$ & No & - \\
\hline OXBE & 0.039 & -0.334 & $(-0.651$ & $-0.016)$ & No & - \\
\hline KIDMED score & 0.040 & 0.301 & $(0.014$ & $0.588)$ & No & - \\
\hline HCB & 0.040 & 0.360 & $(0.017$ & $0.702)$ & No & - \\
\hline Population density (school) & 0.043 & 0.350 & $(0.010$ & $0.690)$ & No & - \\
\hline PFhXS & 0.048 & 0.446 & $(0.005$ & $0.887)$ & No & - \\
\hline
\end{tabular}

${ }^{\text {a }}$ Linear regression models adjusted for cohort, maternal education, maternal age, child age, sex, and trimester of conception. 
Table 3

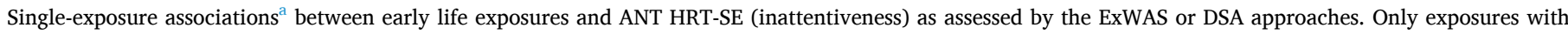
uncorrected p-values $<0.05$ or selected from DSA are shown.

\begin{tabular}{|c|c|c|c|c|c|c|}
\hline & \multicolumn{5}{|l|}{ ExWAS } & \multirow{2}{*}{$\frac{\text { DSA }}{\text { Frequency (\%) of selection }}$} \\
\hline & $\mathbf{p}$ & Adjusted & efficient $(9$ & CI) & Significant after p-value correction? & \\
\hline \multicolumn{5}{|l|}{ Prenatal Exposome } & & \\
\hline Alcohol intake & 0.002 & -14.572 & $(-23.930$ & $-5.214)$ & No & 26 \\
\hline NDVI (100 m) & 0.013 & 15.523 & $(3.247$ & 27.800) & No & 46 \\
\hline DMTP & 0.075 & -5.276 & $(-11.095$ & $0.543)$ & No & 6 \\
\hline \multicolumn{5}{|l|}{ Childhood Exposome } & \multicolumn{2}{|l|}{$\mathrm{TEF}=\mathbf{0 . 0 0 0 7}$} \\
\hline MEHP & 0.006 & 7.800 & $(2.271$ & 13.328) & No & - \\
\hline MiBP & 0.010 & 8.663 & $(2.096$ & $15.230)$ & No & - \\
\hline UV - Vitamin D (day) & 0.015 & 9.260 & $(1.804$ & 16.717) & No & - \\
\hline МEHНP & 0.017 & 7.005 & $(1.243$ & 12.767) & No & - \\
\hline Family affluence score & 0.057 & NA & NA & NA & No & - \\
\hline Middle vs. Low & 0.020 & -17.529 & $(-32.351$ & $-2.707)$ & - & - \\
\hline High vs. Low & 0.125 & -12.049 & $(-27.430$ & $3.332)$ & - & - \\
\hline MEOHP & 0.023 & 6.832 & $(0.964$ & 12.699) & No & - \\
\hline Breastfeeding duration (days) & 0.053 & NA & NA & NA & No & - \\
\hline 2nd tertile $(10.8-34.9)$ vs. 1 st tertile $(<10.8)$ & 0.025 & 12.868 & $(1.591$ & 24.146) & - & - \\
\hline 3rd tertile $(>34.9)$ vs. 1 st tertile $(<10.8)$ & 0.673 & 2.595 & $(-9.477$ & $14.668)$ & - & - \\
\hline Bread intake & 0.029 & NA & NA & NA & No & - \\
\hline 2nd tertile vs. 1 st tertile & 0.221 & 5.992 & $(-3.614$ & 15.599) & - & - \\
\hline 3rd tertile vs. 1 st tertile & 0.080 & -10.178 & $(-21.578$ & $1.222)$ & - & - \\
\hline OH-MiNP & 0.035 & 5.354 & $(0.375$ & 10.333) & No & - \\
\hline Sweets intake & 0.077 & NA & NA & NA & No & - \\
\hline 2nd tertile vs. 1 st tertile & 0.039 & -10.491 & $(-20.472$ & $-0.510)$ & - & - \\
\hline 3rd tertile vs. 1 st tertile & 0.821 & -1.218 & $(-11.785$ & $9.348)$ & - & - \\
\hline DEHP (sum of metabolites) & 0.042 & 6.202 & $(0.223$ & $12.181)$ & No & - \\
\hline Molybdenum & 0.042 & 3.546 & $(0.125$ & $6.967)$ & No & - \\
\hline Walkability index & 0.045 & -6.522 & $(-12.903$ & $-0.142)$ & No & - \\
\hline Meat intake & 0.114 & NA & NA & NA & No & - \\
\hline 2nd tertile vs. 1 st tertile & 0.049 & -10.254 & $(-20.444$ & $-0.064)$ & - & - \\
\hline 3rd tertile vs. 1 st tertile & 0.754 & -1.561 & $(-11.337$ & $8.214)$ & - & - \\
\hline
\end{tabular}

${ }^{\mathrm{a}}$ Linear regression models adjusted for cohort, maternal education, maternal age, child age, sex, and trimester of conception.

Table 4

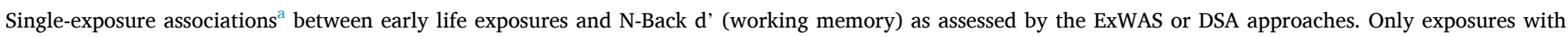
uncorrected p-values $<0.05$ or selected from DSA are shown.

\begin{tabular}{|c|c|c|c|c|c|c|}
\hline & \multicolumn{5}{|l|}{ ExWAS } & \multirow{2}{*}{$\frac{\text { DSA }}{\text { Frequency (\%) of selection }}$} \\
\hline & $\mathrm{p}$ & Adjustec & cient $(95 \%$ & & Significant after p-value correction? & \\
\hline \multicolumn{7}{|l|}{ Prenatal Exposome } \\
\hline Inverse distance to nearest road & 0.017 & 0.101 & $(0.018$ & $0.183)$ & No & - \\
\hline \multicolumn{5}{|l|}{ Childhood Exposome } & \multicolumn{2}{|l|}{$\mathrm{TEF}=\mathbf{0 . 0 0 0 7}$} \\
\hline Organic food intake & 0.006 & NA & NA & NA & No & 12 \\
\hline 2nd tertile vs. 1 st tertile & 0.977 & -0.002 & $(-0.160$ & $0.155)$ & - & - \\
\hline 3rd tertile vs. 1 st tertile & 0.009 & 0.240 & $(0.061$ & $0.418)$ & - & - \\
\hline Indoor PM2.5 & 0.006 & -0.095 & $(-0.164$ & $-0.027)$ & No & 6 \\
\hline DDE & 0.009 & 0.130 & $(0.032$ & $0.227)$ & No & 2 \\
\hline Vegetables intake & 0.038 & NA & NA & NA & No & - \\
\hline 2nd tertile vs. 1 st tertile & 0.011 & 0.219 & $(0.050$ & $0.389)$ & - & - \\
\hline 3rd tertile vs. 1 st tertile & 0.494 & 0.050 & $(-0.093$ & $0.192)$ & - & - \\
\hline KIDMED score & 0.018 & 0.089 & $(0.015$ & $0.163)$ & No & - \\
\hline DDT & 0.020 & 0.124 & $(0.020$ & $0.229)$ & No & - \\
\hline NO2 (year) & 0.026 & -0.220 & $(-0.414$ & $-0.026)$ & No & - \\
\hline $\mathrm{MnBP}$ & 0.027 & -0.097 & $(-0.183$ & $-0.011)$ & No & - \\
\hline Indoor PMabsorbance & 0.036 & -0.073 & $(-0.142$ & $-0.005)$ & No & - \\
\hline Total fat intake & 0.116 & NA & NA & NA & No & - \\
\hline 2nd tertile vs. 1 st tertile & 0.276 & 0.094 & $(-0.075$ & $0.263)$ & - & - \\
\hline 3rd tertile vs. 1 st tertile & 0.039 & 0.192 & $(0.010$ & $0.373)$ & - & - \\
\hline
\end{tabular}

a Linear regression models adjusted for cohort, maternal education, maternal age, child age, sex, and trimester of conception.

regression models excluding the outliers, all with acceptable results (data not shown). We did not find any collinearity between the independent variables, with the exception of child age and cohort site. The collinearity between both variables is moderate and is likely to have little impact on the main findings as strengthened by their concordance with the results stratified by cohort (Supplementary Figures A4.1. and A4.2.).

\section{Discussion}

This is the first study to use a systematic association analysis of many environmental exposures, including lifestyle factors, urban and chemical exposures during pregnancy and childhood periods, and school-agechild neuropsychological development. The study was geographically diverse since six European country-specific birth cohorts participated. Child organic food intake was associated with higher fluid intelligence (CPM) and working memory (N-Back d') scores, and child fast food 

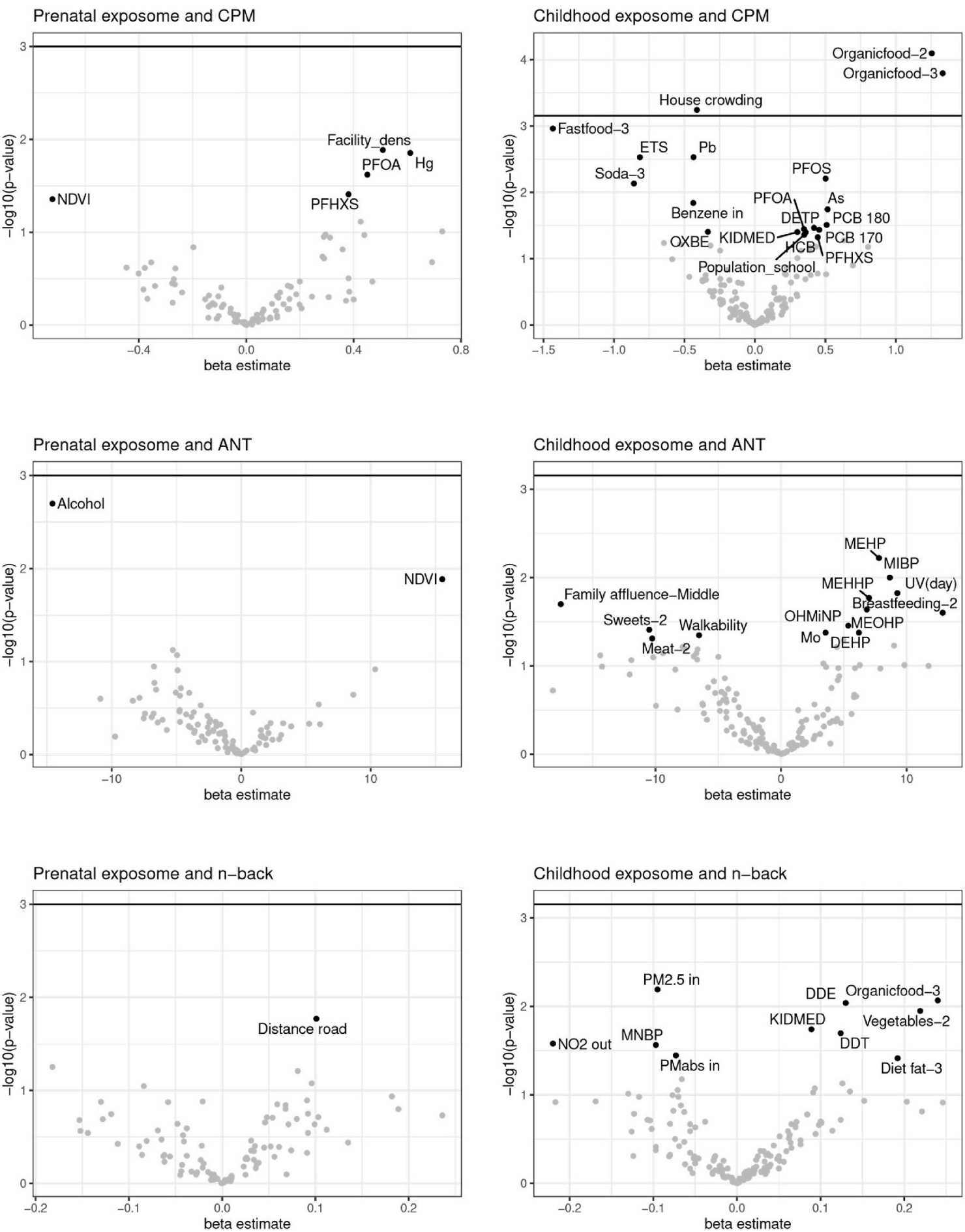

Fig. 1. Exposome-wide associations with child cognitive functions (single-exposure models). On the left panel are the results as volcano plots for the prenatal exposome and the right panel the cross-sectional childhood exposome. The horizontal line across the plots represents the multiple testing threshold correction (based on effective number of tests). Any exposures above this line are considered significant. All the exposures with a p-value below 0.05 are labelled.

intake and house crowding were associated with lower fluid intelligence scores. Child ETS and indoor $\mathrm{PM}_{2.5}$ exposures were associated with lower fluid intelligence and lower working memory scores, respectively. Finally, maternal mercury exposure at higher levels, alcohol consumption, low degree of near-home greenness during pregnancy and child's higher PFOS serum concentration were associated with better fluid intelligence scores or lower inattentiveness (ANT HRT-SE) scores; however, the association directions were unexpected based on previous research of single exposures (Grandjean and Landrigan, 2014).
There are only two previous studies analysing a range of potential risk factors using statistical agnostic methods among cognitive outcomes (Steer et al., 2015; Calamandrei et al., 2020). In one study, the authors used ExWAS to identify prenatal risk factors associated with communication impairments in 9-year-old children from the ALSPAC cohort. They used questionnaire-based data that included health of the parents, child development, and socio-demographic characteristics. Nineteen variables, related to these domain factors were associated with the outcome in the combined multivariable regression model. They detected 
Multiple-exposure associations ${ }^{\mathrm{a}}$ including the exposures selected in at least $5 \%$ of the implementations of DSA.

\begin{tabular}{|c|c|c|c|c|c|c|c|c|c|c|c|c|}
\hline & \multicolumn{4}{|c|}{ CPM (fluid intelligence) } & \multicolumn{4}{|c|}{ ANT HRT-SE (inattentiveness) } & \multicolumn{4}{|c|}{ N-Back d' (working memory) } \\
\hline & \multirow[t]{2}{*}{ Coefficient } & \multicolumn{2}{|l|}{$95 \% \mathrm{CI}$} & \multirow[t]{2}{*}{$\mathrm{p}$} & \multirow[t]{2}{*}{ Coefficient } & \multicolumn{2}{|l|}{$95 \% \mathrm{CI}$} & \multirow[t]{2}{*}{$\mathrm{p}$} & \multirow[t]{2}{*}{ Coefficient } & \multicolumn{2}{|l|}{$95 \% \mathrm{CI}$} & \multirow[t]{2}{*}{$\mathrm{p}$} \\
\hline & & Lower & Upper & & & Lower & Upper & & & Lower & Upper & \\
\hline \multicolumn{13}{|l|}{ Prenatal Exposome } \\
\hline Mercury & 0.611 & $(0.122$ & 1.099) & 0.014 & & & & & & & & \\
\hline Alcohol intake & & & & & -14.925 & $(-24.248$ & $-5.602)$ & 0.002 & & & & \\
\hline NDVI (100 m) & & & & & 16.368 & $(4.153$ & $28.584)$ & 0.009 & & & & \\
\hline DMTP & & & & & -5.168 & $(-10.936$ & $0.600)$ & 0.079 & & & & \\
\hline \multicolumn{13}{|l|}{ Childhood Exposome } \\
\hline $\begin{array}{l}\text { Organic food intake (2nd } \\
\text { tertile) }\end{array}$ & 1.200 & $(0.582$ & 1.817) & $<0.001$ & & & & & -0.011 & $(-0.167$ & $0.146)$ & 0.895 \\
\hline $\begin{array}{l}\text { Organic food intake (3rd } \\
\text { tertile) }\end{array}$ & 1.184 & $(0.502$ & $1.866)$ & 0.001 & & & & & 0.228 & $(0.050$ & $0.406)$ & 0.012 \\
\hline House crowding & -0.392 & $(-0.624$ & $-0.160)$ & 0.001 & & & & & & & & \\
\hline Fastfood intake (2nd tertile) & 0.170 & $(-0.363$ & $0.703)$ & 0.532 & & & & & & & & \\
\hline Fastfood intake (3rd tertile) & -1.250 & $(-2.098$ & $-0.402)$ & 0.004 & & & & & & & & \\
\hline ETS (exposure) & -0.886 & $(-1.419$ & $-0.353)$ & 0.001 & & & & & & & & \\
\hline PFOS & 0.454 & $(0.099$ & $0.808)$ & 0.012 & & & & & & & & \\
\hline Indoor PM2.5 & & & & & & & & & -0.093 & $(-0.161$ & $-0.024)$ & 0.008 \\
\hline
\end{tabular}

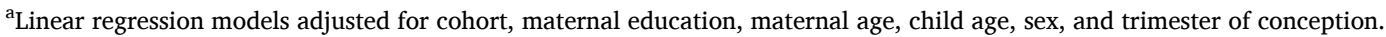

an adverse outcome association with processed food intake during pregnancy. We obtained similar findings with child fast food intake and positive associations with child organic food intake (Steer et al., 2015). In the other study, they used a multiple regression analyses to study the effect of a limited number of prenatal exposome variables, mainly lifestyle factors, and a few metal biomarkers. They found a detrimental effect of lead and, Selenium and mercury, showed positive associations with two-year-old neurodevelopment using Bayley-III Scales (Calamandrei et al., 2020). Furthermore, a recent study based on HELIX project data and child behavioural problems $(n=708)$, using Strengths and Difficulties Questionnaire (SDQ), explored 47 exposure biomarkers from eight chemical families during pregnancy. This study used an adjusted least absolute shrinkage and selection operator (LASSO) and ExWAS and added evidence on deleterious effects of prenatal exposure to bisphenol A (BPA) and mono-n-butyl phthalate (MnBP) on child behavior (Jedynak et al., 2021). More recently, we published a HELIX paper using the same exposome and sub-sample, but in relation to ADHD symptoms and externalizing and internalizing problems based on Child Behavior Check List (CBCL). We observed several prenatal environmental contaminants (indoor air pollution and smoking) and child lifestyle habits (diet, sleep and family social capital) were associated with behavioural problems in children (Maitre et al., 2021). One of the strengths in this previous study on behavioural problems and in our study here on cognition, is that we systematically analysed a much larger range of exposure biomarkers in blood and urine to determine the internal levels in the model, and, we analysed prenatal and childhood exposure variables.

Environmental exposure to methylmercury during pregnancy has been reported to be adversely associated with child cognitive outcomes (Debes et al., 2016). Mercury or methylmercury is considered an important neurotoxic pollutant, particularly during prenatal neurodevelopment (Grandjean and Landrigan, 2014). However, some studies, as ours, reported no adverse associations (Llop et al., 2017). This is probably due to the fact that methylmercury is highly related to fish consumption, which in turn may be negatively confounding the association between methymercury and cognitive scores (Debes et al., 2016; Llop et al., 2017). However, when we adjusted for maternal seafood intake, the results with fluid intelligence scores were unchanged. Prenatal alcohol consumption was associated with lower inattentiveness (low ANT HRT-SE). This finding is not consistent with previous studies (Scott-Goodwin et al., 2016). However, a recent cohort study reported that moderate alcohol intake during pregnancy was not associated with child cognitive development at 12 months (McCormack et al., 2018). Data in the European cohorts of this study, showed that moderate alcohol consumption was more prevalent among highly educated mothers and the reported association was basically driven by this group. Indeed, there may be some misreporting due to social rejection. These two factors could partly explain the un-expected findings described in this study. In relation to greenness exposure, a recent study about life-long exposure (including greenness at birth) found a longitudinal association between higher greenness exposure and lower scores of child inattentiveness (ANT HRT-SE). The association was partly explained by a reduction of ambient air pollution (Dadvand et al., 2017). The inconsistency between our result with inattentiveness (ANT HRT-SE) and this previous finding may be driven by the low maternal education group in some European countries according to our stratified results. Furthermore, this variable does not account for accessibility to green spaces and this factor could mediate the association between the exposure and the outcome, particularly, in poorer neighbourhoods with high greenness exposures (Dadvand et al., 2017; Pearce et al., 2016).

In relation to childhood exposome analyses, there is little previous research specifically analysing child organic food and fast food intakes and cognitive function. However, fast food intake has been associated with a reduction of child academic development success (Purtell and Gershoff, 2015). Furthermore, some studies reported positive associations between children's healthy diet composites and executive function scores (Cohen et al., 2016). Similarly, we found better scores in fluid intelligence and working memory with higher organic food intake and lower fast food intake. Healthy diets (including organic food) are richer than fast food on brain necessary nutrients, such as fatty acids, vitamins and antioxidant substances, and may altogether enhance child cognitive function (Cohen et al., 2016). ETS and home indoor air pollution exposures are recently suspected as potentially hazardous agents affecting cognitive function through enhancing pro-inflammatory reactions in the brain (Grandjean and Landrigan, 2014; Hsieh et al., 2008). The present findings, with the inclusion of participant samples from several European countries, contribute to assume this as a global health concern. House crowding is an indicator of family poverty which includes less healthy life style behaviours than the ones observed in more advantageous social groups, which in turn may affect child cognitive scores (Bitsko et al., 2016). Finally, PFOS is considered an endocrine disruptor that may alter thyroid function and influence cognitive development; however, the epidemiological findings are not consistent (Forns et al., 2015). There may be some potential residual confounding by family social class or education due to some of the exposure sources come from packaged foods and in typical high SES products. Our findings, reporting a positive association between childhood PFOS exposure and cognitive function, add more controversy to this point. 
This study is the first to analyse prenatal and childhood exposome associations with child cognitive outcomes using 'State of the Art' holistic and agnostic statistical approaches on cohort sub-samples of six European countries representing different geographical areas. This region variability adds more external validity or generalizability of the present findings. However, this factor also adds difficulties in our statistical analyses, since participants were from cross-culturally different cohorts, it may influence the exposure levels and cognitive outcomes and the association patterns. Moreover, we reported similar neuropsychological scores to those observed in other population-based studies with children at similar ages, this fact increases the external validity of the present study's findings (Smirni, 2020; Rivas et al., 2019). The statistics applied here may reduce false positive associations (Li et al., 2012), although they can still be present; however, they do not prevent us from potential confounding generated by adverse exposures closely related to beneficial socio-demographic characteristics, neither from reverse causality in childhood exposures, specifically those exposures related with lifestyle factors ${ }^{17,18,19,20}$. Indeed, cross-sectional examination of environmental factors with child cognitive development does not provide an etiologically relevant period of exposure to affect brain development, unless we rely on a strong assumption that exposures were persistent and stable across all childhood. This assumption may be valid in some cases, such as stable life style factors (nutrition) and persistent organic compounds (organochlorine compounds), but less likely in others such as none persistent chemical exposures (bisphenol A, organophosphate pesticides). Also, we should acknowledge that, in the ExWAS and the DSA analyses, we did not consider non-linear associations between exposure and outcome, nor interaction between exposures, to reduce the risk of false positives. However, we had acceptable results, after we tested the linearity of the multi-exposure associations by plotting the residuals versus the predicted values and we repeated the regression models excluding the outliers. Finally, the statistical power of this study was limited, nevertheless, the power calculation showed sufficient results for this sample size.

A strength of this study is its originality to explore a large range of human exposures and select the main cognitive determinants based on data driven approach. Furthermore, our approach of reporting all associations is more transparent than usual studies, which may be affected by selective reporting of results. It also shows the complexity of the human exposome and that the association with child cognitive development is challenging. Furthermore, we need innovative scientific approaches in order to understand its nature.

For the first time in this study, we analysed systematically many prenatal and childhood exposures (including chemical biomarkers) and their association with school-age neuropsychological development (as child's cognitive functioning). The findings describe a priority list of exposures susceptible to be strongly associated with European child cognitive functioning. This is clinically important for future environmental interventions to target specific risk factors of mental health development. Child organic and fast food diets, family crowdedness, indoor air pollution and ETS were the main determinants of fluid intelligence and/or working memory. However, a limitation is that these associations are cross-sectional and confounding and reverse causation cannot be plenty avoided. Furthermore, the conflicting results observed with other exposures, such as prenatal mercury, home greenness, maternal alcohol consumption and child PFOS levels need further investigation in order to understand their complexity in the association patterns.

\section{Contributors' statements}

This study was conceived and designed by Martine Vrijheid in collaboration with Jordi Julvez. The analysis was conducted by Mónica López Vicente. A first draft of the manuscript was prepared by Jordi Julvez. Charline Warembourg, Lea Maitre, Claire Philippat, Kristine B. Gützkow, Monica Guxens and Jorunn Evandt participated in the interpretation of data and drafting of the manuscript for important intellectual content, Xavier Basagaña, Remy Slama participated in the statistical design and protocol, and reviewed the manuscript for important intellectual content. Montserrat de Castro, David DonaireGonzález, Maribel Casas, Carles Hernandez-Ferrer, Mark Nieuwenhuijsen, Oliver Robinson, Ibon Tamayo-Uria, Jose Urquiza participated in designing data collection protocols and data managing and curation, and reviewed the manuscript for important intellectual content. Sandra Andrusaityte, Miguel Burgaleta, Lida Chatzi, Regina Gražulevičiene, Barbara Heude, Rosie Mceachan, Mark Mon-Williams, Amrit K. Sakhi, Nuria Sebastian-Galles, Jordi Sunyer, Cathrine Thomsen, Marina Vafeiadi and John Wright FRCP, participated in the review the manuscript for important intellectual content.

\section{Funding}

This publication reflects only the author's views and the European Commission is not liable for any use that may be made of the information contained therein. The research leading to these results has received funding from the European Community's Seventh Framework Programme (FP7/2007-206) under grant agreement no 308333 - the HELIX project. The LifeCycle project, involved in this study, received funding from the European Union's Horizon 2020 research and innovation programme (Grant Agreement No. 733206 LifeCycle). This study has been funded by Instituto de Salud Carlos III through the projects "CP14/00108 \& PI16/00261" (Co-funded by European Regional Development Fund "A way to make Europe"). Jordi Julvez held a Miguel Servet contract (MS14/00108) awarded by the Spanish Institute of Health Carlos III, Ministry of Economy and Competitiveness). Jordi Julvez holds Miguel Servet-II contract (CPII19/00015) awarded by the Instituto de Salud Carlos III (Co-funded by European Social Fund "Investing in your future"). CW holds a Sara Borrell postdoctoral grant (CD18/00132) from the Instituto de Salud Carlos III. MC received funding from Instituto de Salud Carlos III, Ministry of Economy and Competitiveness) (MS16/00128). Project "PI16/00118", funded by Instituto de Salud Carlos III and co-funded by European Union (FEDER) "A way to make Europe". INMA data collections were supported by grants from the Instituto de Salud Carlos III, CIBERESP, and the Generalitat de Catalunya- CIRIT (Spain). KANC was funded by the grant of the Lithuanian Agency for Science Innovation and Technology (6-042014_31V-66). The Norwegian Mother, Father and Child Cohort Study is supported by the Norwegian Ministry of Health and Care Services and the Ministry of Education and Research. The Rhea project was financially supported by European projects, and the Greek Ministry of Health (Program of Prevention of obesity and neurodevelopmental disorders in preschool children, in Heraklion district, Crete, Greece: 2011-2014; "Rhea Plus": Primary Prevention Program of Environmental Risk Factors for Reproductive Health, and Child Health: 2012-15). The work was also supported by MICINN [MTM2015-68140-R] and Centro Nacional de Genotipado- CEGEN- PRB2- ISCIII (Spain). This paper presents independent research funded by the National Institute for Health Research (NIHR) under its Collaboration for Applied Health Research and Care (CLAHRC) for Yorkshire and Humber (UK). Core support for Born in Bradford is also provided by the Wellcome Trust (WT101597MA, UK). The LIFE-CYCLE project has received funding from the European Union's Horizon 2020 research and innovation programme under grant agreement No 733206. The EDEN study was supported by Foundation for medical research (FRM), National Agency for Research (ANR), National Institute for Research in Public health (IRESP: TGIR cohorte santé 2008 program), French Ministry of Health (DGS), French Ministry of Research, INSERM Bone and Joint Diseases National Research (PRO-A), and Human Nutrition National Research Programs, Paris-Sud University, Nestlé, French National Institute for Population Health Surveillance (InVS), French National Institute for Health Education (INPES), the European Union FP7 programmes (FP7/2007-2013, HELIX, ESCAPE, ENRIECO, Medall projects), Diabetes National Research Program 
(through a collaboration with the French Association of Diabetic Patients (AFD)), French Agency for Environmental Health Safety (now ANSES), Mutuelle Générale de l'Education Nationale a complementary health insurance (MGEN), French national agency for food security, French-speaking association for the study of diabetes and metabolism (ALFEDIAM).

ISGlobal is a member of the Agency for the Research Centres of Catalonia (CERCA) Programme, Generalitat de Catalunya. We are grateful to all the participating children, parents, practitioners and researchers in the six countries who took part in this study.

\section{Financial disclosure}

Authors have no financial relationships relevant to this article to 31 disclose.

\section{Declaration of competing interest}

The authors declare that they have no known competing financial interests or personal relationships that could have appeared to influence the work reported in this paper.

\section{Acknowledgments}

We further thank Muireann Coen, Sonia Brishoual, Angelique Serre, Michele Grosdenier, Prof Frederic Millot, Elodie Migault, Manuela Boue, Sandy Bertin, Veronique Ferrand-Rigalleau, Céline Leger, Noella Gorry, Silvia Fochs, Nuria Pey, Cecilia Persavento, Susana Gross, Georgia Chalkiadaki, Danai Feida, Eirini Michalaki, Mariza Kampouri, Anny Kyriklaki, Minas Iakovidis, Maria Fasoulaki, Ingvild Essen, Heidi Marie Nordheim, and the Yorkshire Water. The authors thank the EDEN mother-child cohort study group, whose members are I. AnnesiMaesano, J.Y. Bernard, J. Botton, M.A. Charles, P. Dargent-Molina, B. de Lauzon-Guillain, P. Ducimetière, M. de Agostini, B. Foliguet, A. Forhan, X. Fritel, A. Germa, V. Goua, R. Hankard, B. Heude, M. Kaminski, B. Larroquey, N. Lelong, J. Lepeule, G. Magnin, L. Marchand, C. Nabet, F Pierre, R. Slama, M.J. Saurel-Cubizolles, M. Schweitzer, and O. Thiebaugeorges.

\section{Appendix A. Supplementary data}

Supplementary data to this article can be found online at https://doi. org/10.1016/j.envpol.2021.117404.

\section{References}

Agier, L., Portengen, L., Chadeau-Hyam, M., Basagaña, X., Giorgis-Allemand, L., Siroux, V., Robinson, O., Vlaanderen, J., González, J.R., Nieuwenhuijsen, M.J., Vineis, P., Vrijheid, M., Slama, R., Vermeulen, R., 2016. A systematic comparison of linear regression-based statistical methods to assess exposome-health associations. Environ. Health Perspect. 124 (12), 1848-1856.

Bitsko, R.H., Holbrook, J.R., Robinson, L.R., Kaminski, J.W., Ghandour, R., Smith, C. Peacock, G., 2016. Health Care, family, and community factors associated with mental, behavioral, and developmental disorders in early childhood - United States, 2011-2012. MMWR Morb. Mortal. Wkly. Rep. 65 (9), 221-226.

Braun, J.M., Hoffman, E., Schwartz, J., Sanchez, B., Schnaas, L., Mercado-Garcia, A. Solano-Gonzalez, M., Bellinger, D.C., Lanphear, B.P., Hu, H., Tellez-Rojo, M.M., Wright, R.O., Hernandez-Avila, M., 2012. Assessing windows of susceptibility to lead-induced cognitive deficits in Mexican children. Neurotoxicology 33 (5), 1040-1047.

Braun, J.M., Muckle, G., Arbuckle, T., Bouchard, M.F., Fraser, W.D., Ouellet, E., Séguin, J.R., Oulhote, Y., Webster, G.M., Lanphear, B.P., 2017. Associations of prenatal urinary bisphenol A concentrations with child behaviors and cognitive abilities. Environ. Health Perspect. 125 (6), 067008.

Calamandrei, G., Ricceri, L., Meccia, E., Tartaglione, A.M., Horvat, M., Tratnik, J.S., Mazej, D., Špirić, Z., Prpić, I., Vlašić-Cicvarić, I., Neubauer, D., Kodrič, J., Stropnik, S., Janasik, B., Kuraś, R., Mirabella, F., Polańska, K., Chiarotti, F., 2020. Pregnancy exposome and child psychomotor development in three European birth cohorts. Environ. Res. 181, 108856.

Cohen, J.F., Gorski, M.T., Gruber, S.A., Kurdziel, L.B., Rimm, E.B., 2016. The effect of healthy dietary consumption on executive cognitive functioning in children and adolescents: a systematic review. Br. J. Nutr. 116 (6), 989-1000.
Dadvand, P., Tischer, C., Estarlich, M., Llop, S., Dalmau-Bueno, A., López-Vicente, M., Valentín, A., de Keijzer, C., Fernández-Somoano, A., Lertxundi, N., RodriguezDehli, C., Gascon, M., Guxens, M., Zugna, D., Basagaña, X., Nieuwenhuijsen, M.J., Ibarluzea, J., Ballester, F., Sunyer, J., 2017. Lifelong residential exposure to green space and attention: a population-based prospective study. Environ. Health Perspect. 125 (9), 097016.

Debes, F., Weihe, P., Grandjean, P., 2016. Cognitive deficits at age 22 years associated with prenatal exposure to methylmercury. Cortex 74, 358-369.

Forns, J., Lertxundi, N., Aranbarri, A., Murcia, M., Gascon, M., Martinez, D., Grellier, J., Lertxundi, A., Julvez, J., Fano, E., Goñi, F., Grimalt, J.O., Ballester, F., Sunyer, J., Ibarluzea, J., 2012. Prenatal exposure to organochlorine compounds and neuropsychological development up to two years of life. Environ. Int. 45, 72-77.

Forns, J., Esnaola, M., López-Vicente, M., Suades-González, E., Alvarez-Pedrerol, M., Julvez, J., et al., 2014. The n-back test and the attentional network task as measures of child neuropsychological development in epidemiological studies. Neuropsychology 28 (4), 519-529.

Forns, J., Iszatt, N., White, R.A., Mandal, S., Sabaredzovic, A., Lamoree, M., Thomsen, C., Haug, L.S., Stigum, H., Eggesbø, M., 2015. Perfluoroalkyl substances measured in breast milk and child neuropsychological development in a Norwegian birth cohort study. Environ. Int. 83, 176-182.

Grandjean, P., Landrigan, P.J., 2014. Neurobehavioural effects of developmental toxicity. Lancet Neurol. 13 (3), 330-338.

Haug, L.S., Sakhi, A.K., Cequier, E., Casas, M., Maitre, L., Basagana, X., Andrusaityte, S., Chalkiadaki, G., Chatzi, L., Coen, M., de Bont, J., Dedele, A., Ferrand, J., Grazuleviciene, R., Gonzalez, J.R., Gutzkow, K.B., Keun, H., McEachan, R., Meltzer, H.M., Petraviciene, I., Robinson, O., Saulnier, P.J., Slama, R., Sunyer, J., Urquiza, J., Vafeiadi, M., Wright, J., Vrijheid, M., Thomsen, C., 2018. In-utero and childhood chemical exposome in six European mother-child cohorts. Environ. Int. 121 (Pt 1), 751-763.

Heyer, D.B., Meredith, R.M., 2017. Environmental toxicology: sensitive periods of development and neurodevelopmental disorders. Neurotoxicology 58, 23-41.

Hsieh, C.J., Liao, H.F., Wu, K.Y., Hsieh, W.S., Su, Y.N., Jeng, S.F., Yu, S.N., Chen, P.C., 2008. CYP1A1 Ile462Val and GSTT1 modify the effect of cord blood cotinine on neurodevelopment at 2 years of age. Neurotoxicology 29 (5), 839-845.

Jedynak, P., Maitre, L., Guxens, M., Gützkow, K.B., Julvez, J., López-Vicente, M., Sunyer, J., Casas, M., Chatzi, L., Gražulevičienè, R., Kampouri, M., McEachan, R., Mon-Williams, M., Tamayo, I., Thomsen, C., Urquiza, J., Vafeiadi, M., Wright, J., Basagaña, X., Vrijheid, M., Philippat, C., 2021 Apr 1. Prenatal exposure to a wide range of environmental chemicals and child behaviour between 3 and 7 years of age - an exposome-based approach in 5 European cohorts. Sci. Total Environ. 763, 144115. https://doi.org/10.1016/j.scitotenv.2020.144115.

Jin, Y., Hein, M.J., Deddens, J.A., Hines, C.J., 2011. Analysis of lognormally distributed exposure data with repeated measures and values below the limit of detection using SAS. Ann. Occup. Hyg. 55, 97-112.

Lee, E.H., Chung, B.Y., Suh, C.H., Jung, J.Y., 2015. Korean versions of the Perceived Stress Scale (PSS-14, 10 and 4): psychometric evaluation in patients with chronic disease. Scand. J. Caring Sci. 29 (1), 183-192.

Li, M.X., Yeung, J.M., Cherny, S.S., Sham, P.C., 2012. Evaluating the effective numbers of independent tests and significant p-value thresholds in commercial genotyping arrays and public imputation reference datasets. Hum. Genet. 131 (5), 747-756.

Llop, S., Ballester, F., Murcia, M., Forns, J., Tardon, A., Andiarena, A., Vioque, J. Ibarluzea, J., Fernández-Somoano, A., Sunyer, J., Julvez, J., Rebagliato, M., LopezEspinosa, M.J., 2017. Prenatal exposure to mercury and neuropsychological development in young children: the role of fish consumption. Int. J. Epidemiol. 46 (3), 827-838.

Maitre, L., de Bont, J., Casas, M., et al., 2018. Human Early Life Exposome (HELIX) study: a European population-based exposome cohort. BMJ Open, e021311. https://doi. org/10.1136/bmjopen-2017-021311.

Maitre, L., Julvez, J., López-Vicente, M., Warembourg, C., Tamayo-Uria, I., Philippat, C., Gützkow, K.B., Guxens, M., Andrusaityte, S., Basagaña, X., Casas, M., de Castro, M., Chatzi, L., Evandt, J., Gonzalez, J.R., Gražulevičienè, R., Smastuen Haug, L., Heude, B., Hernandez-Ferrer, C., Kampouri, M., Manson, D., Marquez, S., McEachan, R., Nieuwenhuijsen, M., Robinson, O., Slama, R., Thomsen, C., Urquiza, J., Vafeidi, M., Wright, J., Vrijheid, M., 2021 Mar 25. Early-life environmental exposure determinants of child behavior in Europe: a longitudinal, population-based study. Environ. Int. 153, 106523. https://doi.org/10.1016/j. envint.2021.106523.

McCormack, C., Hutchinson, D., Burns, L., Youssef, G., Wilson, J., Elliott, E., Allsop, S., Najman, J., Jacobs, S., Rossen, L., Olsson, C., Mattick, R., 2018. Maternal and partner prenatal alcohol use and infant cognitive development. Drug Alcohol Depend. 185, 330-338.

Nieuwenhuijsen, M.J., Smith, R., Golfinopoulos, S., Best, N., Bennett, J., Aggazzotti, G., Righi, E., Fantuzzi, G., Bucchini, L., Cordier, S., Villanueva, C.M., Moreno, V., La Vecchia, C., Bosetti, C., Vartiainen, T., Rautiu, R., Toledano, M., Iszatt, N., Grazuleviciene, R., Kogevinas, M., 2009. Health impacts of long-term exposure to disinfection by-products in drinking water in Europe: hiwate. J. Water Health 7 (2), 185-207.

Olesen, T.S., Bleses, D., Andersen, H.R., Grandjean, P., Frederiksen, H., Trecca, F., Bilenberg, N., Kyhl, H.B., Dalsager, L., Jensen, I.K., Andersson, A.M., Jensen, T.K. 2017. Prenatal phthalate exposure and language development in toddlers from the Odense Child Cohort. Neurotoxicol. Teratol. 65, 34-41.

Pearce, A., Sawyer, A.C.P., Chittleborough, C.R., Mittinty, M.N., Law, C., Lynch, J.W., 2016. Do early life cognitive ability and self-regulation skills explain socio-economic inequalities in academic achievement? An effect decomposition analysis in UK and Australian cohorts. Soc. Sci. Med. 165, 108-118. 
Purtell, K.M., Gershoff, E.T., 2015. Fast food consumption and academic growth in late childhood. Clin Pediatr (Phila). 54 (9), 871-877.

Raven, J., 1998. Raven Coloured Progressive Matrices Manual. Harcourt assessment. J C Raven and J H Raven, San Antonio. Texas. USA.

Rivas, I., Basagaña, X., Cirach, M., López-Vicente, M., Suades-González, E., GarciaEsteban, R., Álvarez-Pedrerol, M., Dadvand, P., Sunyer, J., 2019. Association between early life exposure to air pollution and working memory and attention. Environ. Health Perspect. 127 (5), 57002.

Robinson, O., Tamayo, I., de Castro, M., Valentin, A., Giorgis-Allemand, L., Hjertager Krog, N., Marit Aasvang, G., Ambros, A., Ballester, F., Bird, P., Chatzi, L., Cirach, M., Dedele, A., Donaire-Gonzalez, D., Gražuleviciene, R., Iakovidis, M., Ibarluzea, J., Kampouri, M., Lepeule, J., Maitre, L., McEachan, R., Oftedal, B., Siroux, V., Slama, R., Stephanou, E.G., Sunyer, J., Urquiza, J., Vegard Weyde, K., Wright, J., Vrijheid, M., Nieuwenhuijsen, M., Basagaña, X., 2018. The urban exposome during pregnancy and its socioeconomic determinants. Environ. Health Perspect. 126 (7), 077005.

Roze, E., Meijer, L., Bakker, A., Van Braeckel, K.N., Sauer, P.J., Bos, A.F., 2009. Prenatal exposure to organohalogens, including brominated flame retardants, influences motor, cognitive, and behavioral performance at school age. Environ. Health Perspect. 117 (12), 1953-1958.

Scott-Goodwin, A.C., Puerto, M., Moreno, I., 2016. Toxic effects of prenatal exposure to alcohol, tobacco and other drugs. Reprod. Toxicol. 61, 120-130.

Sinisi, S.E., van der Laan, M.J., 2004. Deletion/substitution/addition algorithm in learning with applications in genomics. Stat. Appl. Genet. Mol. Biol. 3.

Smirni, D., 2020. The raven's coloured progressive Matrices in healthy children: a qualitative approach. Brain Sci. 10 (11), 877.

Steer, C.D., Bolton, P., Golding, J., 2015. Preconception and prenatal environmental factors associated with communication impairments in 9 year old children using an exposome-wide approach. Plos One 10 (3), e0118701.
Sunyer, J., Esnaola, M., Alvarez-Pedrerol, M., Forns, J., Rivas, I., López-Vicente, M., Suades-González, E., Foraster, M., Garcia-Esteban, R., Basagaña, X., Viana, M., Cirach, M., Moreno, T., Alastuey, A., Sebastian-Galles, N., Nieuwenhuijsen, M., Querol, X., 2015. Association between traffic-related air pollution in schools and cognitive development in primary school children: a prospective cohort study. PLoS Med. 12 (3), e1001792.

Tamayo-Uria, I., Maitre, L., Thomsen, C., Nieuwenhuijsen, M.J., Chatzi, L., Siroux, V., Aasvang, G.M., Agier, L., Andrusaityte, S., Casas, M., de Castro, M., Dedele, A.,

Haug, L.S., Heude, B., Grazuleviciene, R., Gutzkow, K.B., Krog, N.H., Mason, D., McEachan, R.R.C., Meltzer, H.M., Petraviciene, I., Robinson, O., Roumeliotaki, T., Sakhi, A.K., Urquiza, J., Vafeiadi, M., Waiblinger, D., Warembourg, C., Wright, J., Slama, R., Vrijheid, M., Basagaña, X., 2019. The early-life exposome: description and patterns in six European countries. Environ. Int. 123, 189-200.

Textor, J., Hardt, J., Knüppel, S., 2011. DAGitty: a graphical tool for analyzing causal diagrams. Epidemiology 22 (5), 745.

Vrijheid, M., et al., 2014. The human early-life exposome (HELIX): project rationale and design. Environ. Health Perspect. 122 (6), 535-544.

van Wendel de Joode, B., Mora, A.M., Lindh, C.H., Hernández-Bonilla, D., Córdoba, L., Wesseling, C., Hoppin, J.A., Mergler, D., 2016. Pesticide exposure and neurodevelopment in children aged 6-9 years from Talamanca, Costa Rica. Cortex 85, 137-150.

Weyde, K.V., Krog, N.H., Oftedal, B., Magnus, P., Øverland, S., Stansfeld, S., Nieuwenhuijsen, M.J., Vrijheid, M., de Castro Pascual, M., Aasvang, G.M., 2017. Road traffic noise and children's inattention. Environ. Health 16 (1), 127.

White, I.R., Royston, P., Wood, A.M., 2011. Multiple imputation using chained equations: issues and guidance for practice. Stat. Med. 30 (4), 377-399. 